\title{
Several Dynamic Properties of Solutions to a Generalized Camassa-Holm Equation
}

\author{
Zheng Yin \\ Department of Mathematics, Southwestern University of Finance and Economics, Chengdu 610074, China \\ Correspondence should be addressed to Zheng Yin; yinzheng@swufe.edu.cn
}

Received 31 March 2013; Accepted 12 May 2013

Academic Editor: Shaoyong Lai

Copyright (c) 2013 Zheng Yin. This is an open access article distributed under the Creative Commons Attribution License, which permits unrestricted use, distribution, and reproduction in any medium, provided the original work is properly cited.

For a nonlinear generalization of the Camassa-Holm equation, we investigate the dynamic properties of solutions for the equation under the assumption that the initial value $u_{0}(x)$ lies in the space $H^{1}(R)$. A one-sided upper bound estimate on the first-order spatial derivative, $L^{p}$ bound estimate, and a space-time higher-norm estimate for the solutions are obtained.

\section{Introduction}

Hakkaev and Kirchev [1] investigated the following generalized Camassa-Holm equation:

$$
u_{t}-u_{t x x}+\left(2 k u+\frac{n+2}{2} u^{n+1}\right)_{x}=\left(n u^{n-1} \frac{u_{x}^{2}}{2}+u^{n} u_{x x}\right)_{x}
$$

where $n$ is an integer. When $n=1$, (1) becomes the Camassa-Holm model (see [2]). The local well-posedness in the Sobolev space $H^{s}$ with $s>3 / 2$ is established, and sufficient conditions for the stability and instability of the solitary wave solutions are given in [1]. However, the $L^{p}$ estimate of strong solutions and one-sided upper bound estimate on the first-order spatial derivative for the solutions are not discussed in [1]. This constitutes the objective of this work.

Like the Camassa-Holm equation (see $[1,2])$, (1) has the conservation law

$$
\int_{R}\left(u^{2}(t, x)+u_{x}^{2}(t, x)\right) d x=\int_{R}\left(u^{2}(0, x)+u_{x}^{2}(0, x)\right) d x
$$

which plays an important role in our further investigations.

In fact, many scholars have paid their attentions to the study of the Camassa-Holm equation. The existence of global weak solutions is established in Constantin and Escher [3],
Constantin and Molinet [4], Xin and Zhang [5], and Coclite et al. [6]. It was shown in Constantin and Escher [7] that the blowup occurs in the form of breaking waves. Namely, the solution remains bounded, but its slope becomes unbounded in finite time. After wave breaking, the solution is continued uniquely either as a global conservative weak solution $[8,9]$ or as a global dissipative solution $[10,11]$. Exact traveling wave solutions for the Camassa-Holm equation are presented in [12]. For other methods to investigate the problems involving various dynamic properties of the Camassa-Holm equation, the reader is referred to [13-16] and the references therein.

In this paper, we investigate several dynamic properties of strong solutions for the generalized Camassa-Holm equation (1) in the case where $n$ is an odd natural number and the assumption $u_{0}(x) \in H^{1}(R)$. The results obtained in this work include a one-sided upper bound estimate on the firstorder derivatives of the solution, a space-time higher-norm estimate, and the $L^{p}(2 \leq p<\infty)$ bound estimate.

The rest of this paper is organized as follows. Section 2 states the main result. Several lemmas are given in Section 3 where the proof of main result is completed.

\section{Main Result}

Let $m$ be a nonnegative integer and $n=2 m+1$. In this case, the Cauchy problem for (1) is written in the form

$$
u_{t}-u_{t x x}+\partial_{x}\left(2 k u+\frac{2 m+3}{2} u^{2 m+2}\right)
$$




$$
\begin{aligned}
= & \frac{1}{2 m+2} u^{2 m+2} \partial_{x}^{3} u^{2 m+2} \\
& -\frac{2 m+1}{2} \partial_{x}\left(u^{2 m} u_{x}^{2}\right), \\
u(0, x) & =u_{0}(x)
\end{aligned}
$$

which is equivalent to

$$
\begin{gathered}
u_{t}+u^{2 m+1} u_{x}+\frac{\partial H}{\partial x}=0, \\
\frac{\partial H}{\partial x}=\Lambda^{-2} \partial_{x}\left[2 k u+\frac{2 m^{2}+5 m+2}{2(m+1)} u^{2 m+2}+\frac{2 m+1}{2} u^{2 m} u_{x}^{2}\right] \\
u(0, x)=u_{0}(x),
\end{gathered}
$$

where the operator $\Lambda^{2}=1-\partial^{2} / \partial x^{2}$ and

$$
\Lambda^{-2} f(x)=\frac{1}{2} \int_{R} e^{-|x-y|} f(y) d y \quad \text { for } f(x) \in L^{2}(R) .
$$

We introduce a result presented in [1] for problem (3).

Lemma 1 (see [1]). Suppose that $u_{0} \in H^{s}$ with constant $s>$ $3 / 2$. Then there is a real number $T>0$ such that the problem (3) has a unique solution $u(t, x)$ satisfying

$$
u(t, x) \in C\left([0, T) ; H^{s}(R)\right) \bigcap C^{1}\left([0, T) ; H^{s-1}(R)\right) .
$$

Now we state the main result of this paper.

Theorem 2. Let $u_{0} \in H^{s}(R)$ with $s>3$. Then the solution of problem (3) has the following properties.

(a) There exists a positive constant $c_{0}$ depending on $m$ and $\left\|u_{0}\right\|_{H^{1}(R)}$ such that the one-sided $L^{\infty}$ norm estimate on the first-order spatial derivative holds

$$
\frac{\partial u(t, x)}{\partial x} \leq c_{0}(1+t), \quad \text { for }(t, x) \in[0, \infty) \times R .
$$

(b) Let $0<\gamma<1, T>0$, and $a, b \in R, a<b$. Then there exists a positive constant $c_{1}$ depending only on $\left\|u_{0}\right\|_{H^{1}(R)}, \gamma, T, a, b$, and $m$ such that the following estimate holds:

$$
\int_{0}^{t} \int_{a}^{b} u^{2 m}\left|\frac{\partial u(t, x)}{\partial x}\right|^{2+\gamma} d x \leq c_{1}
$$

(c) There exists a constant $c_{2}$ depending only on $\left\|u_{0}\right\|_{H^{1}(R)}$, and $m$, p such that

$$
\|u\|_{L^{p}(R)} \leq c_{2}(1+t), \quad t \in[0, \infty), 2 \leq p<\infty .
$$

\section{Proof of Main Result}

From the conservation law (2), we have

$$
\|u\|_{L^{\infty}(R)} \leq\|u\|_{H^{1}(R)}=\left\|u_{0}\right\|_{H^{1}(R)} .
$$

Differentiating the first equation of problem (4) with respect to $x$ and writing $\partial u / \partial x=q$, we obtain

$$
\begin{aligned}
\frac{\partial q}{\partial t}+ & u^{2 m+1} \frac{\partial q}{\partial x}+\frac{2 m+1}{2} u^{2 m} q^{2} \\
= & 2 k u+\frac{2 m^{2}+5 m+2}{2(m+1)} u^{2 m+2} \\
& -\Lambda^{-2}\left[2 k u+\frac{2 m^{2}+5 m+2}{2(m+1)} u^{2 m+2}+\frac{2 m+1}{2} u^{2 m} q^{2}\right] \\
= & Q(t, x) .
\end{aligned}
$$

Lemma 3. Let $0<\gamma<1, T>0$, and $a, b \in R, a<$ $b$. Then there exists a positive constant $c_{1}$ depending only on $\left\|u_{0}\right\|_{H^{1}(R)}, \gamma, T, a, b$, and $m$, such that the space higher integrability estimate holds

$$
\int_{0}^{T} \int_{a}^{b} u^{2 m}\left|\frac{\partial u(t, x)}{\partial x}\right|^{2+\gamma} d x \leq c_{1}
$$

where $u=u(t, x)$ is the unique solution of problem (3).

Proof. The proof is a variant of the proof presented in Xin and Zhang [5] (or see Coclite et al. [6]). Let $\phi \in C^{\infty}(R)$ be a cut-off function such that $0<\phi<1$ and

$$
\phi(x)= \begin{cases}1, & \text { if } x \in[a, b] \\ 0, & \text { if } x \in(-\infty, a-1] \cup[b+1, \infty)\end{cases}
$$

Letting $\theta(\xi)=\xi(1+|\xi|)^{\gamma}, \xi \in R$, and $0<\gamma<1$ yields

$$
\begin{aligned}
\theta^{\prime}(\xi)= & (1+(1+\gamma)|\xi|)(1+|\xi|)^{\gamma-1}, \theta^{\prime \prime}(\xi) \\
= & \gamma \operatorname{sign}(\xi)(1+|\xi|)^{\gamma-2}(2+(1+\gamma)|\xi|) \\
= & \gamma(1+\gamma) \operatorname{sign}(\xi)(1+|\xi|)^{\gamma-1} \\
& +(1-\gamma) \gamma \operatorname{sign}(\xi)(1+|\xi|)^{\gamma-2},
\end{aligned}
$$

from which we get

$$
\begin{aligned}
|\theta(\xi)| \leq|\xi|+|\xi|^{1+\gamma}, \quad\left|\theta^{\prime}(\xi)\right| \leq 1+(1+\gamma)|\xi| & \left|\theta^{\prime \prime}(\xi)\right| \leq 2 \gamma \\
\xi \theta(\xi)-\frac{1}{2} \xi^{2} \theta^{\prime}(\xi) & =\frac{1-\gamma}{2} \xi^{2}(1+|\xi|)^{\gamma}+\frac{\gamma}{2} \xi^{2}(1+|\xi|)^{\gamma-1} \\
& \geq \frac{1-\gamma}{2} \xi^{2}(1+|\xi|)^{\gamma} .
\end{aligned}
$$


Multiplying (11) by $\phi \theta^{\prime}(q)$, using the chain rule, and integrating over $\pi_{T}:=[0, T] \times R$, we have

$$
\begin{aligned}
& (2 m+1) \int_{\pi_{T}} \phi(x) u^{2 m} q \theta(q) d t d x \\
& -\frac{2 m+1}{2} \int_{\pi_{T}} \phi(x) u^{2 m} q^{2} \theta^{\prime}(q) d t d x \\
& =\int_{R} \phi(x)(\theta(q(t, x))-\theta(q(0, x))) d x \\
& -\int_{\pi_{T}} u^{2 m+1} \phi^{\prime}(x) \theta(q) d t d x \\
& -\int_{\pi_{T}} Q(t, x) \phi(x) \theta^{\prime}(q) d t d x .
\end{aligned}
$$

From (16), we get

$$
\begin{aligned}
& (2 m+1) \int_{\pi_{T}} \phi(x) u^{2 m} q \theta(q) d t d x \\
& \quad-\frac{2 m+1}{2} \int_{\pi_{T}} \phi(x) u^{2 m} q^{2} \theta^{\prime}(q) d t d x \\
& =\int_{\pi_{T}}(2 m+1) \phi(x) u^{2 m}\left(q \theta(q)-\frac{1}{2} q^{2} \theta^{\prime}(q)\right) d t d x \\
& \geq \frac{(1-\gamma)}{2} \int_{\pi_{T}} \phi(x) u^{2 m} q^{2}(1+|q|)^{\gamma} d t d x .
\end{aligned}
$$

Using the Hölder inequality, (2), and (15) yields

$$
\begin{aligned}
& \left|\int_{R} \phi(x) \theta(q) d x\right| \\
& \leq \int_{R} \phi(x)\left(|q|^{1+\gamma}+|q|\right) d x \\
& \leq\|\phi\|_{L^{2 /(1-\gamma)(R)}}\|q\|_{L^{2}(R)}^{1+\gamma}+\|\phi\|_{L^{2}(R)}\|q\|_{L^{2}(R)} \\
& \leq(b-a+2)^{(1-\gamma) / 2}\left\|u_{0}\right\|_{H^{1}(R)}^{1+\gamma}+(b-a+2)^{1 / 2}\left\|u_{0}\right\|_{H^{1}(R)}, \\
& \left|\int_{\pi_{T}} u^{2 m+1} \phi^{\prime}(x) \theta(q) d t d x\right| \\
& \leq \int_{\pi_{T}}|u|^{2 m+1}\left|\phi^{\prime}(x)\right|\left(|q|^{1+\gamma}+|q|\right) d t d x \\
& \leq\left\|u_{0}\right\|_{H^{1}(R)}^{12 m+1} \int_{0}^{T}\left(\left\|\phi^{\prime}\right\|_{L^{2 /(1-\gamma)}(R)}\|q\|_{L^{2}(R)}^{1+\gamma}\right. \\
& \left.\quad+\left\|\phi^{\prime}\right\|_{L^{2}(R)}\|q\|_{L^{2}(R)}\right) d t \\
& \leq c T\left(\left\|\phi^{\prime}\right\|_{L^{2 /(1-\gamma)}(R)}\left\|u_{0}\right\|_{H^{1}(R)}^{1+\gamma}+\left\|\phi^{\prime}\right\|_{L^{2}(R)}\left\|u_{0}\right\|_{H^{1}(R)}\right) .
\end{aligned}
$$

Using (10), we obtain

$$
\begin{gathered}
\left\|2 k u+\frac{2 m^{2}+5 m+2}{2(m+1)} u^{2 m+2}\right\|_{L^{\infty}} \leq c, \\
\left\|\Lambda^{-2}\left[2 k u+\frac{2 m^{2}+5 m+2}{2(m+1)} u^{2 m+2}\right]\right\|_{L^{\infty}} \leq c .
\end{gathered}
$$

Applying (10), the Hölder inequality, and $\int_{R} e^{-|x-y|} d y=2$, we have

$$
\left|\Lambda^{-2}\left[u^{2 m} q^{2}\right]\right|=\left|\int_{R} \frac{1}{2} e^{-|x-y|} u^{2 m} u_{y}^{2} d y\right| \leq c\|u\|_{H^{1}(R)} \leq c,
$$

where $c$ is a constant depending on $\left\|u_{0}\right\|_{H^{1}(R)}$ and $m$.

From (20) and (21), we deduce that there exists a positive constant $c$ depending on $\left\|u_{0}\right\|_{H^{1}(R)}$ and $m$, such that

$$
\|Q(t, x)\|_{L^{\infty}(R)} \leq c,
$$

from which we get

$$
\begin{aligned}
& \left|\int_{\pi_{T}} Q(t, x) \phi(x) \theta^{\prime}(q) d t d x\right| \\
& \leq c \int_{\pi_{T}}|\phi(x)|((1+\gamma)|q|+1) d t d x \\
& \leq c T\left((1+\gamma)\|\phi(x)\|_{L^{2}(R)}\left\|u_{0}\right\|_{H^{1}(R)}\right. \\
& \left.+\int_{R}|\phi(x)| d x\right) .
\end{aligned}
$$

From inequalities (18)-(19) and (23), we obtain (12).

Lemma 4. There exists a positive constant $c$ depending only on $\left\|u_{0}\right\|_{H^{1}(R)}$ and $m$ such that

$$
\|Q(t, \cdot)\|_{L^{\infty}(R)} \leq c, \quad\left\|\frac{\partial H(t, \cdot)}{\partial x}\right\|_{L^{\infty}(R)} \leq c .
$$

If $k=0$, it holds that

$$
\begin{array}{ll}
\|Q(t, \cdot)\|_{L^{1}(R)} \leq c, & \left\|\frac{\partial H(t, \cdot)}{\partial x}\right\|_{L^{1}(R)} \leq c, \\
\|Q(t, \cdot)\|_{L^{2}(R)} \leq c, & \left\|\frac{\partial H(t, \cdot)}{\partial x}\right\|_{L^{2}(R)} \leq c .
\end{array}
$$

Proof. We have

$$
\begin{aligned}
Q(t, x) & \\
= & 2 k u+\frac{2 m^{2}+5 m+2}{2(m+1)} u^{2 m+2} \\
& -\Lambda^{-2}\left[2 k u+\frac{2 m^{2}+5 m+2}{2(m+1)} u^{2 m+2}+\frac{2 m+1}{2} u^{2 m} q^{2}\right], \\
\frac{\partial H}{\partial x}= & \Lambda^{-2} \partial_{x}\left[2 k u+\frac{2 m^{2}+5 m+2}{2(m+1)} u^{2 m+2}+\frac{2 m+1}{2} u^{2 m} u_{x}^{2}\right] .
\end{aligned}
$$


The first inequality of (24) is proved in Lemma 3 (see (22)). For the second inequality in (24), we have

$$
\begin{aligned}
& \left|\frac{\partial H(t, \cdot)}{\partial x}\right| \\
& =\mid \frac{1}{2} \int_{R} e^{-|x-y|} \\
& \times\left[2 k u+\frac{2 m^{2}+5 m+2}{2(m+1)} u^{2 m+2}\right. \\
& \left.+\frac{2 m+1}{2} u^{2 m} u_{y}^{2}\right]_{y} d y \\
& =\mid \frac{1}{2} e^{-x} \int_{-\infty}^{x} e^{y}\left[2 k u+\frac{2 m^{2}+5 m+2}{2(m+1)} u^{2 m+2}\right. \\
& \left.+\frac{2 m+1}{2} u^{2 m} u_{y}^{2}\right]_{y} d y \\
& +\frac{1}{2} e^{x} \int_{x}^{\infty} e^{-y}\left[2 k u+\frac{2 m^{2}+5 m+2}{2(m+1)} u^{2 m+2}\right. \\
& \left.+\frac{2 m+1}{2} u^{2 m} u_{y}^{2}\right]_{y} d y \\
& =\mid-\frac{1}{2} e^{-x} \int_{-\infty}^{x} e^{y}\left[2 k u+\frac{2 m^{2}+5 m+2}{2(m+1)} u^{2 m+2}\right. \\
& \left.+\frac{2 m+1}{2} u^{2 m} u_{y}^{2}\right] d y \\
& +\frac{1}{2} e^{x} \int_{x}^{\infty} e^{-y}\left[2 k u+\frac{2 m^{2}+5 m+2}{2(m+1)} u^{2 m+2}\right. \\
& \left.+\frac{2 m+1}{2} u^{2 m} u_{y}^{2}\right] d y \\
& \leq \int_{R} e^{-|x-y|} \mid 2 k u+\frac{2 m^{2}+5 m+2}{2(m+1)} u^{2 m+2} \\
& +\frac{2 m+1}{2} u^{2 m} u_{y}^{2} \mid d y \\
& \leq c\|u\|_{H^{1}(R)} \\
& \leq c\left\|u_{0}\right\|_{H^{1}(R)},
\end{aligned}
$$

which together with (22) results in (24).

In fact, we have

$$
\begin{gathered}
\left\|u^{2 m+2}\right\|_{L^{1}(R)} \leq c\|u\|_{L^{2}(R)}^{2} \leq c\|u\|_{H^{1}(R)}^{2} \leq c\left\|u_{0}\right\|_{H^{1}(R)}^{2}, \\
\left\|\Lambda^{-2}\left[\frac{2 m^{2}+5 m+2}{2(m+1)} u^{2 m+2}+\frac{2 m+1}{2} u^{2 m} q^{2}\right]\right\|_{L^{1}(R)} \\
\leq c\|u\|_{H^{1}(R)}^{2} \leq c\left\|u_{0}\right\|_{H^{1}(R)}^{2},
\end{gathered}
$$

$$
\begin{aligned}
\left\|\Lambda^{-2}\left[\partial_{x} u^{2 m+2}\right]\right\|_{L^{1}(R)} & \leq \iint_{R} e^{-|x-y|} u^{2 m+2} d x d y \\
& \leq c\|u\|_{H^{1}(R)}^{2} \leq c\left\|u_{0}\right\|_{H^{1}(R)}^{2}, \\
\left\|\Lambda^{-2} \partial_{x}\left[u^{2 m} u_{x}^{2}\right]\right\|_{L^{1}(R)} & \leq \iint_{R} e^{-|x-y|} u^{2 m} u_{y}^{2} d x d y \\
& \leq c\|u\|_{H^{1}(R)}^{2} \leq c\left\|u_{0}\right\|_{H^{1}(R)^{\prime}}^{2} .
\end{aligned}
$$

From (3)-(31), we obtain (25).

Using

$$
\begin{gathered}
\|Q(t, \cdot)\|_{L^{2}(R)}^{2} \leq\|Q(t, \cdot)\|_{L^{\infty}}\|Q(t, \cdot)\|_{L^{1}(R)} \leq c, \\
\left\|\frac{\partial H(t, \cdot)}{\partial x}\right\|_{L^{2}(R)}^{2} \leq\left\|\frac{\partial H(t, \cdot)}{\partial x}\right\|_{L^{\infty}}\left\|\frac{\partial H(t, \cdot)}{\partial x}\right\|_{L^{1}(R)} \leq c
\end{gathered}
$$

completes the proof of (26).

From Lemma 1, we know that for any $u_{0} \in H^{s}(R)$ with $s>3 / 2$, there exist a maximal $T=T\left(u_{0}\right)>0$ and a unique strong solution $u$ to problem (3) such that

$$
u \in C\left([0, T) ; H^{s}(R)\right) C^{1}\left([0, T) ; H^{s-1}(R)\right) .
$$

Consider the differential equation

$$
\begin{gathered}
\xi_{t}=u^{2 m+1}(t, \xi), \quad t \in[0, T), \\
\xi(0, x)=x .
\end{gathered}
$$

Lemma 5. Assume $u_{0} \in H^{s}, s>3$, and let $T>0$ be the maximal existence time of the solution to problem (3). Then there exists a unique solution $\xi \in C^{1}([0, T) \times R, R)$ to problem (31). In addition, the map $p(t, \cdot)$ is an increasing diffeomorphism of $R$ with $\xi_{x}(t, x)>0$ for $(t, x) \in[0, T) \times R$.

Proof. Using Lemma 1, we obtain $u \in C^{1}\left([0, T) ; H^{s-1}(R)\right)$ and $H^{s-1} \in C^{1}(R)$. Therefore, we know that functions $u(t, x)$ and $u_{x}(t, x)$ are bounded, Lipschitz in space, and $C^{1}$ in time. The existence and uniqueness theorem for differential equations guarantees that problem (31) has a unique solution $\xi \epsilon$ $C^{1}([0, T) \times R, R)$.

From (31), we get

$$
\begin{gathered}
\frac{d}{d t} \xi_{x}=(2 m+1) u^{2 m} u_{\xi}(t, \xi) \xi_{x}, \quad t \in[0, T), \\
p_{x}(0, x)=1
\end{gathered}
$$

Furthermore,

$$
\xi_{x}(t, x)=\exp \left(\int_{0}^{t}(2 m+1) u^{2 m} u_{\xi}(\tau, \xi(\tau, x)) d \tau\right) .
$$

For every $T^{\prime}<T$, the Sobolev imbedding theorem gives rise to

$$
\sup _{(\tau, x) \in\left[0, T^{\prime}\right) \times R}\left|u_{x}(\tau, x)\right|<\infty .
$$

Therefore, there exists a constant $K_{0}>0$ such that $\xi_{x}(t, x) \geq$ $e^{-K_{0} t}$ for $(t, x) \in[0, T) \times R$. The proof is completed. 
Using (11) and (32), we get

$$
\begin{aligned}
\frac{d u_{\xi}(t, \xi(t, x))}{d t} & =\frac{d u_{\xi}(t, \xi(t, x))}{d t}+\frac{d u_{\xi}(t, \xi(t, x))}{d \xi} \xi_{t} \\
& =\frac{d u_{\xi}(t, \xi(t, x))}{d t}+\frac{d u_{\xi}(t, \xi(t, x))}{d \xi} u^{2 m+1} \\
& =Q(t, \xi(t, x))-\frac{2 m+1}{2} u^{2 m} q^{2} \\
& \leq\|Q(t, x)\|_{L^{\infty}} \\
& \leq c .
\end{aligned}
$$

It follows from (35) that

$$
u_{\xi}(t, \xi(t, x)) \leq c+c t
$$

Using Lemma 5 and (36), we have

$$
u_{x}(t, x) \leq c+c t, \quad t \in[0, \infty)
$$

Using the first equation of problem (4) and Lemma 4, for an arbitrary integer $K \geq 2$, we have

$$
\begin{aligned}
& \frac{d}{d t} \int_{R} u^{2 K} d x \\
& \quad=2 K \int_{R} u^{2 K-1} \frac{d u}{d t} d x \\
& \quad=2 K \int_{R} u^{2 K-1}\left[-u^{2 m+1} u_{x}-\frac{\partial H}{\partial x}\right] d x \\
& \quad=-2 K \int_{R} u^{2 K+2 m} u_{x} d x-2 K \int_{R} u^{2 K-1} \frac{\partial H}{\partial x} d x \\
& \quad=-2 K \int_{R} u^{2 K-1} \frac{\partial H}{\partial x} d x \\
& \quad \leq 2 K\left\|_{L^{\infty}(R)}^{2 K-3}\right\| \frac{\partial H}{\partial x}\left\|_{L^{\infty}(R)}\right\| u \|_{L^{2}(R)}^{2} \\
& \leq c\left\|u_{0}\right\|_{H^{1}(R)} \\
& \leq c
\end{aligned}
$$

from which we get

$$
\int_{R} u^{2 K} d x \leq c t+\int_{R} u_{0}^{2 K} d x \leq c t+c\left\|u_{0}\right\|_{L^{\infty}(R)}\left\|u_{0}\right\|_{H^{1}(R)}^{2} .
$$

By the $L^{p}$ interpolation theorem, for all $2 \leq p<\infty$, we obtain

$$
\|u\|_{L^{p}(R)} \leq c(1+t), \quad t \in[0, \infty),
$$

where $c$ depends on $\left\|u_{0}\right\|_{H^{1}(R)}$ and $p$.

From Lemma 3 and (37) and (40), we complete the proof of Theorem 2.

\section{Acknowledgment}

This work is supported by the Fundamental Research Funds for the Central Universities (JBK120504).

\section{References}

[1] S. Hakkaev and K. Kirchev, "Local well-posedness and orbital stability of solitary wave solutions for the generalized CamassaHolm equation," Communications in Partial Differential Equations, vol. 30, no. 4-6, pp. 761-781, 2005.

[2] R. Camassa and D. D. Holm, "An integrable shallow water equation with peaked solitons," Physical Review Letters, vol. 71, no. 11, pp. 1661-1664, 1993.

[3] A. Constantin and J. Escher, "Global weak solutions for a shallow water equation," Indiana University Mathematics Journal, vol. 47, no. 4, pp. 1527-1545, 1998.

[4] A. Constantin and L. Molinet, "Global weak solutions for a shallow water equation," Communications in Mathematical Physics, vol. 211, no. 1, pp. 45-61, 2000.

[5] Z. Xin and P. Zhang, "On the weak solutions to a shallow water equation," Communications on Pure and Applied Mathematics, vol. 53, no. 11, pp. 1411-1433, 2000.

[6] G. M. Coclite, H. Holden, and K. H. Karlsen, "Global weak solutions to a generalized hyperelastic-rod wave equation," SIAM Journal on Mathematical Analysis, vol. 37, no. 4, pp. 10441069,2005

[7] A. Constantin and J. Escher, "Wave breaking for nonlinear nonlocal shallow water equations," Acta Mathematica, vol. 181, no. 2, pp. 229-243, 1998.

[8] A. Bressan and A. Constantin, "Global conservative solutions of the Camassa-Holm equation," Archive for Rational Mechanics and Analysis, vol. 183, no. 2, pp. 215-239, 2007.

[9] H. Holden and X. Raynaud, "Global conservative solutions of the Camassa-Holm equation-a Lagrangian point of view," Communications in Partial Differential Equations, vol. 32, no. 10-12, pp. 1511-1549, 2007.

[10] O. G. Mustafa, "Global dissipative solutions of the generalized Camassa-Holm equation," Journal of Nonlinear Mathematical Physics, vol. 15, no. 1, pp. 96-115, 2008.

[11] H. Holden and X. Raynaud, "Global conservative solutions of the generalized hyperelastic-rod wave equation," Journal of Differential Equations, vol. 233, no. 2, pp. 448-484, 2007.

[12] H.-H. Dai, "Exact travelling-wave solutions of an integrable equation arising in hyperelastic rods," Wave Motion, vol. 28, no. 4, pp. 367-381, 1998.

[13] S. Lai and Y. Wu, "The local well-posedness and existence of weak solutions for a generalized Camassa-Holm equation," Journal of Differential Equations, vol. 248, no. 8, pp. 2038-2063, 2010.

[14] S. Lai and Y. Wu, "Global solutions and blow-up phenomena to a shallow water equation," Journal of Differential Equations, vol. 249, no. 3, pp. 693-706, 2010.

[15] S. Lai and A. Wang, "The well-posedness of solutions for a generalized shallow water wave equation," Abstract and Applied Analysis, vol. 2012, Article ID 872187, 15 pages, 2012.

[16] S. Y. Lai, "The global weak solution for a generalized CamassaHolm equation," Abstract and Applied Analysis, vol. 2013, Article ID 838302, 6 pages, 2013 


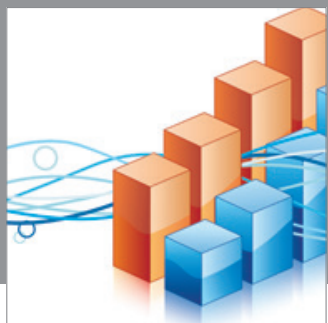

Advances in

Operations Research

mansans

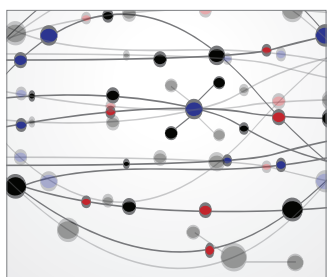

The Scientific World Journal
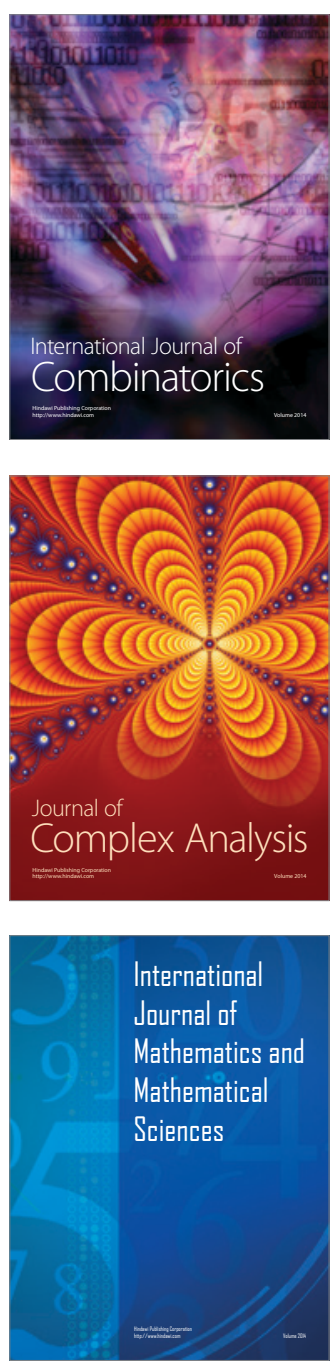
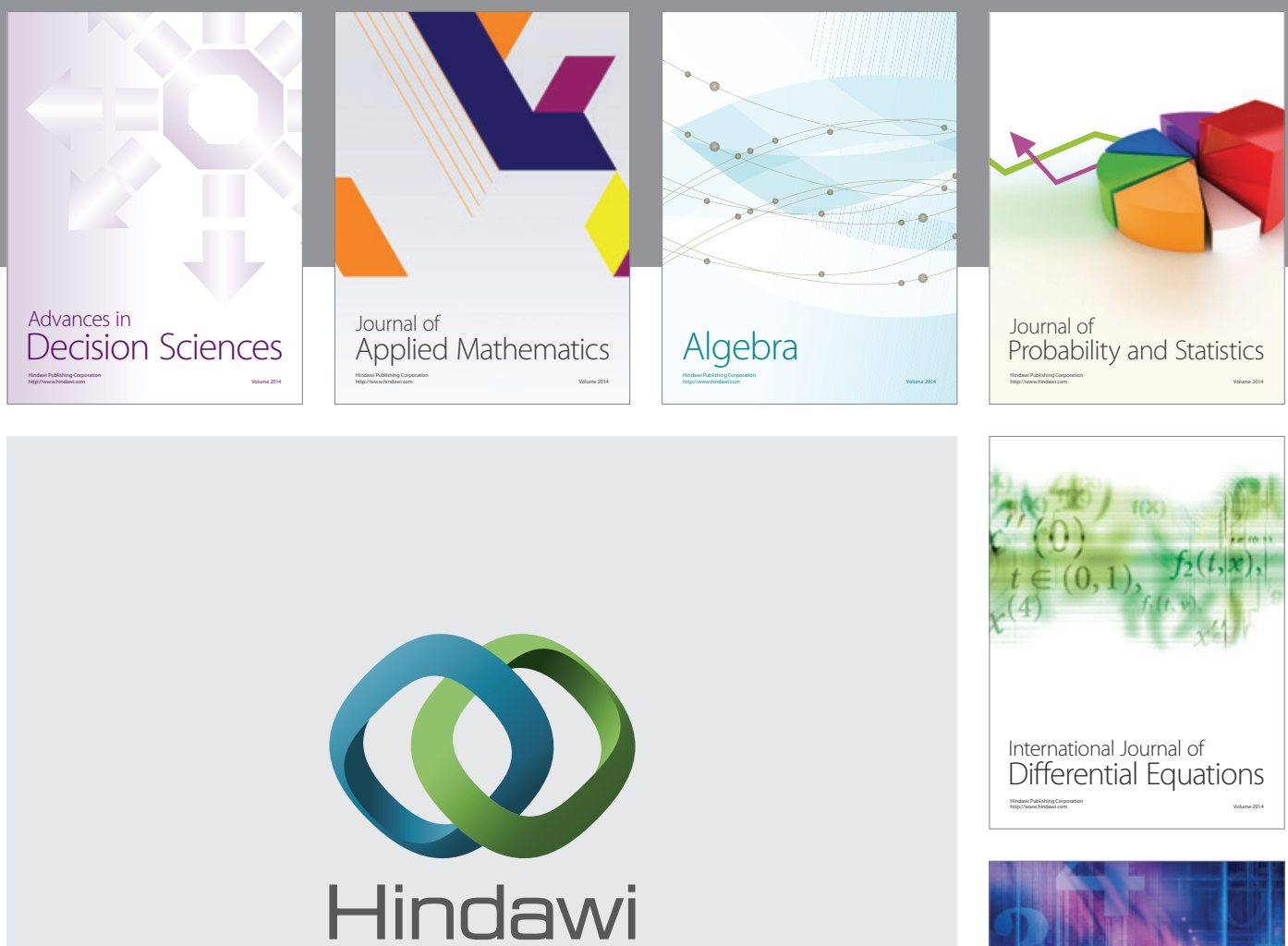

Submit your manuscripts at http://www.hindawi.com
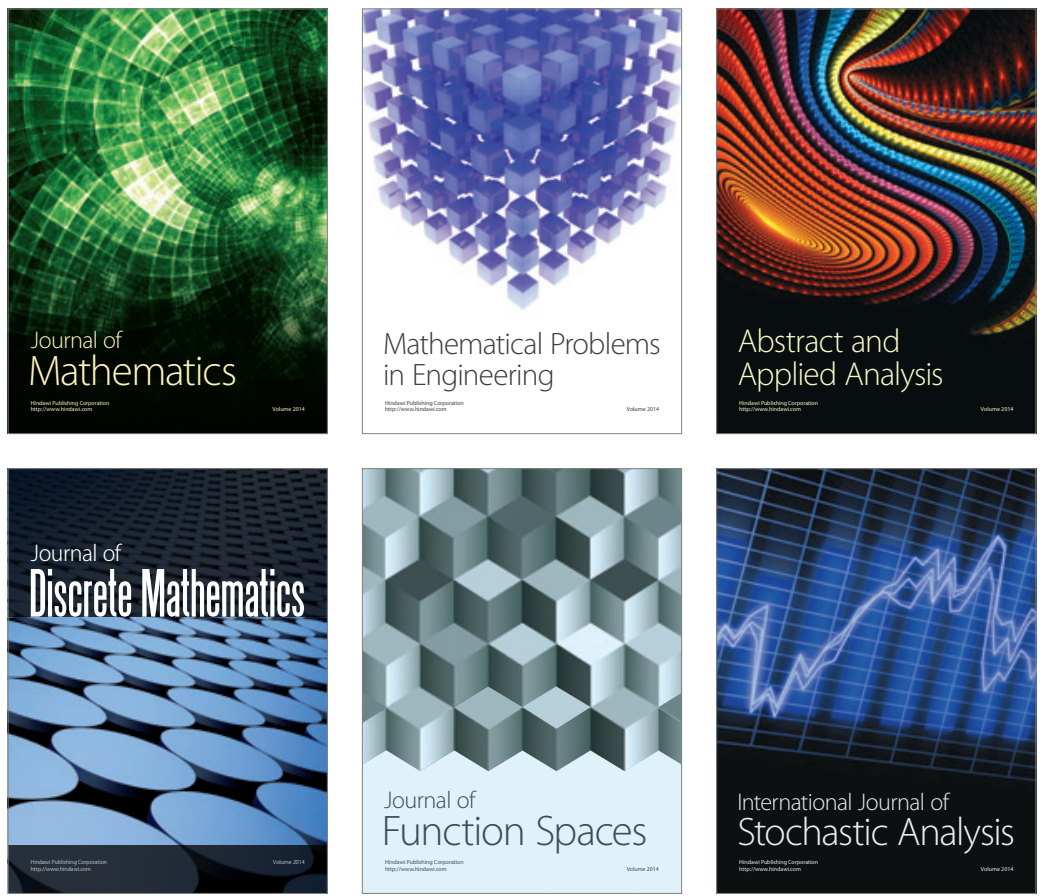

Journal of

Function Spaces

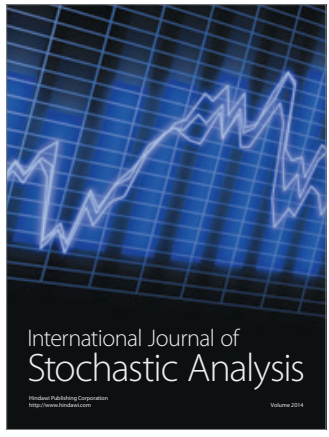

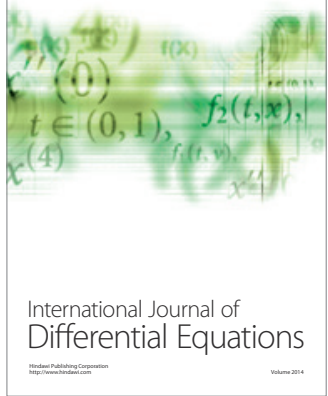
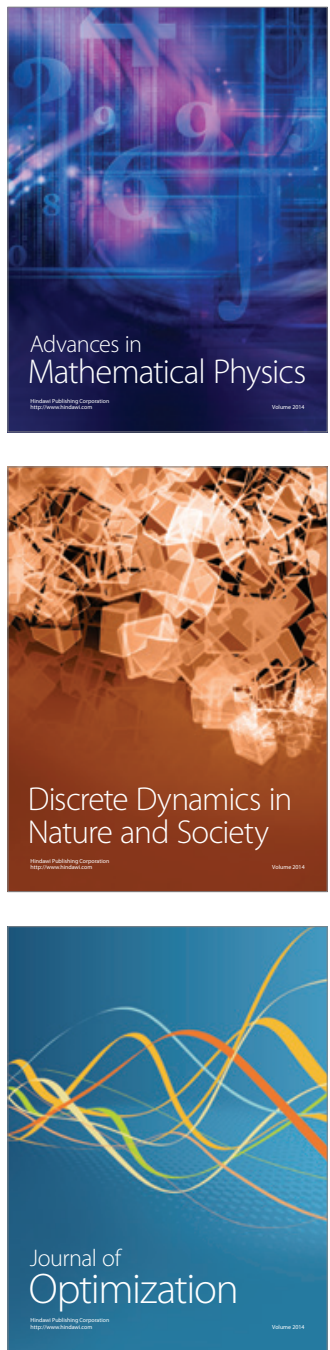